\title{
A APLICAÇÃO DO LEAN MANUFACTURING NA LINHA DE PRODUÇÃO DO BISCOITO TORRADINHA ALHO
}

\author{
Antônio Elízio de Oliveira - antonio.oliveira@professor.unis.edu.br \\ Felipe Xavier Ribeiro Pacifico - fxpacifico@hotmail.com \\ Gilberto Sousa Lopes- gilberto.lopes@professor.unis.edu.br \\ Letícia da Silva Silvano - leticiasilvano55@gmail.com \\ Paulo Henrique Lopes - paulohenrique022@hotmail.com
}

\section{RESUMO}

Atualmente a competitividade na indústria alimentícia vem aumentando gradativamente, fazendo com que as empresas sofram pressões para aprimorar o meio produtivo, buscando melhorias dos processos. O estudo tem como objetivo a aplicação do Lean Manuacturing na linha de produção do biscoito torradinha alho, na busca por melhorias e otimização de processos. Onde no estado atual a produção é realizada de forma não sincronizada e sem padronização, assim, havendo movimentação desnecessária de funcionários por distanciamento das células de produção, empilhamento de tabuleiros e interrupção no processo para coleta de materiais. Com aplicação da metodologia a empresa obteve melhorias no processo, sendo capaz de reduzir sua movimentação em $37 \%$, e ainda assim, conseguindo alcançar mais uma redução de $1,58 \%$ no custo geral da produção e o aumento de 155 quilos de produto acabado.

Palavras-chave: Processo produtivo. Biscoito torradinha alho. Lean Manufacturing

\section{INTRODUÇÃO}

Com a competitividade as empresas estão em busca de métodos e técnicas que reduzam custos operacionais e visam agregar melhorias no produto acabado para disponibilizar mercadorias de alta qualidade e com bons preços para o mercado alavancando suas vendas. Para isso é necessário adotar estratégicas na aplicação de métodos e técnicas que otimizem a produção. Diante disso, é possível citar o sistema de produção enxuta, que é o 
sistema Toyota de Produção, mais conhecido como Lean Manufacturing. Método que visa descartar tudo aquilo que não agrega valor para o cliente e levar agilidade aos processos, ou seja, busca eliminar perdas e desperdícios. O Sistema Toyota de Produção, tem como um dos seus objetivos a otimização de recursos, buscando sempre produzir mais gastando menos.

O estudo foi desenvolvido na linha de produção do biscoito torradinha alho por ser o biscoito mais vendido nos últimos quatro anos e por ser um processo que possui muitas etapas como: preparação, modelagem, empilhamento de tabuleiros, encaixe de tabuleiros no carrinho de assadura, $1^{\mathrm{a}}$ assadura, corte, tempero, empilhamento de tabuleiros, encaixe de tabuleiros no carrinho para segunda etapa de assadura, $2^{\mathrm{a}}$ assadura, desinformação, pesagem, embalagem e expedição, onde requer grande número de funcionários para execução dessas tarefas.

O trabalho tem como objetivo a redução de custos e aumento de produtividade no setor do biscoito torradinha alho, para oferecer um produto com muita qualidade e melhor preço, assim, alavancando suas vendas.Oestado atual a produção é realizada de forma não sincronizada e sem padronização, assim, havendo movimentação desnecessária de funcionários por distanciamento das células de produção, empilhamento de tabuleiros, interrupção no processo para coleta de materiais.Onde os objetivos específicos são:

- Estudar layout para aproximação das células de produção;

- Eliminar etapa de empilhamento de tabuleiros;

- Eliminar etapa de recolhimento de tabuleiros nas bancadas;

- Cronometrar atividades para levantamento de custo de produção;

- Redução de movimentação;

- Redução de custo de produção.

Após definição dos objetivos, foi realizado a implementação do Lean Manufacturingem busca de melhorias para o processo produtivo.

\section{REVISÃO DE LITERATURA}

\subsection{Lean Manufacturing}

De acordo com Arunagiri e Gnanavelbabu (2014), o Lean Manufacturing é um método que visa descartar tudo aquilo que não agrega valor para o cliente e levar agilidade 
aos processos, ou seja, busca eliminar perdas e desperdícios. O Sistema Toyota de Produção, tem como um dos seus objetivos a otimização de recursos, buscando sempre produzir mais gastando menos, e por isso foi denominado um sistema Lean Manufacturing.

Para Ohno(1997), quando falamos sobre o Lean Manufecturing é importante entender as perdas do sistema de produção enxuta. A base do Sistema Toyota de Produção é a eliminação de desperdícios.

\subsection{Perdas segundo o Sistema Toyota de Produção}

De acordo com Ohno (1997), vinculada a produção enxuta possuem perdas durante um processo.No desenvolvimento do trabalho foram identificadas 2 perdas em seu processo produtivo, sendo elas:

\subsubsection{Movimentação}

Para Ohno (1997), os percursos realizados pelos colaboradores envolvidos no processo acarretam esse tipo de perda. Quanto mais próxima a movimentação feita pelo funcionário durante a execução da atividade e que não o afete ergonomicamente ou a segurança dele, melhor para empresa. Portanto toda atividade realizada deve agregar valor ao processo.

\subsubsection{Processamento}

Essa perda é diretamente ligada a atividades desnecessárias durante o processo. Tais “atividades desnecessárias" são partes do processamento que podem ser eliminadas sem danificar características e/ou funções básicas do produto ou serviço. (Ohno, 1997)

\section{METODOLOGIA}

Para o desenvolvimento do presente artigo foram realizadas pesquisas bibliográficas através de revistas e artigos científicos no período de junho de 2019 a junho de 2020. 
A proposta do estudo apresenta o avanço do processo através da aplicação do Lean Manufacturingno setor de produção do biscoito torradinha alho, assim como embasado de forma exploratória o objetivo de mostrar o desenvolvimento completo do projeto, e sua perspectiva de cenário primário e cenário futuro.

O artigo foi desenvolvido através de referencial teórico e aplicação do Lean Manufacturing na linha de produção da Indústria e Comércio de Biscoitos Amanteigados $\mathrm{N}^{\circ} 1$. O estudo foi realizado através da coleta de dados do processo produtivo e cronoanálise das atividades desenvolvidas.

\subsection{Cronoanálise}

De acordo com Linhar e Luzzatto (2011), a cronoanálise é uma ferramenta para determinar tempos operacionais e atividades para análise de processos produtivos. A cronoanálise foi utilizada para obter o tempo exercido pelos operadores nas atividades do processo produtivo no cenário primário e em seguida no secundário, no intuito de demonstrar melhorias no processo com a aplicação da metodologia.

\subsection{Software Microsoft Excel}

Para Bezerra (2019), a ferramenta possui estrutura capaz de gerar planilhas com dados e elaborações de gráficos. O Excel foi utilizado para elaboração das tabelas de análise de custo de produção e movimentação.

\subsection{Diagrama de espaguete}

Segundo Freitas (2013), o diagrama de espaguete é um método utilizado para identificar e apresentar a movimentação de um processo produtivo, onde tem como principais etapas: delimitação da área, observação, desenhar o fluxo, definir o fluxo e identificar oportunidades de melhorias. O diagrama teve como objetivo demonstrar o caminho percorrido pelos operadores durante o processo produtivo antes e depois da alteração de layout.

\subsection{Software AutoCAD}


Segundo Castro (2010), o programa é utilizado para projetos em até três dimensões, possibilitando o estudo e a criação de formas e volumes em diversos setores. O softwarefoi utilizado para construção da área de produção na elaboração do diagrama de espaguete.

\subsection{Software Microsoft Paint}

Para Bezerra (2019), a ferramenta da Microsoft para elaboração de desenhos e edição de imagens. Foi utilizado para desenhar o trajeto percorrido pelos operadores durante o processo produtivo.

\subsection{Software Arena}

De acordo com Prado (1999), o software possui um conjunto de módulos onde poder ser utilizados para simular e descrever processos produtivos. A ferramenta foi utilizada para simulação das etapas de produção.

\section{7 $\quad$ Empresa de aplicação da ferramenta}

$\mathrm{O}$ estudo foi realizado na Indústria e Comercio de Biscoitos $\mathrm{N}^{\circ} 1$, localizada na Avenida Manoel Inácio Peixoto ,1101, Bairro: Industrial, na Cidade de Cataguases, Minas Gerais. Fundada em 1996, é uma Empresa de pequeno porte, que tem como atividade principal a fabricação de biscoitos amanteigados. Possui 40 colaboradores no setor de produção, fabricando mais de 70 sabores em toda sua linha de produção.

\section{RESULTADOS E DISCUSSÕES}

Com base no mapeamento do processo, identificou-se a possibilidade da redução de perdas existentesno processo produtivo. Mediante o diagrama de espaguete, foi possível identificar a distância percorrida pelos operadoresdurante a movimentação. Observa-se na figura 01construída no AutoCAD e o percurso demonstrado pelo Paint, que os operadores envolvidos no processo percorriam 24.312 metros, onde o ponto crítico está na distância 
percorrida pelo Forneiro e o Confeiteiro, que são responsáveis pelas movimentações entre as etapas produtivas.

Figura01:Diagrama de espaguete cenário primário

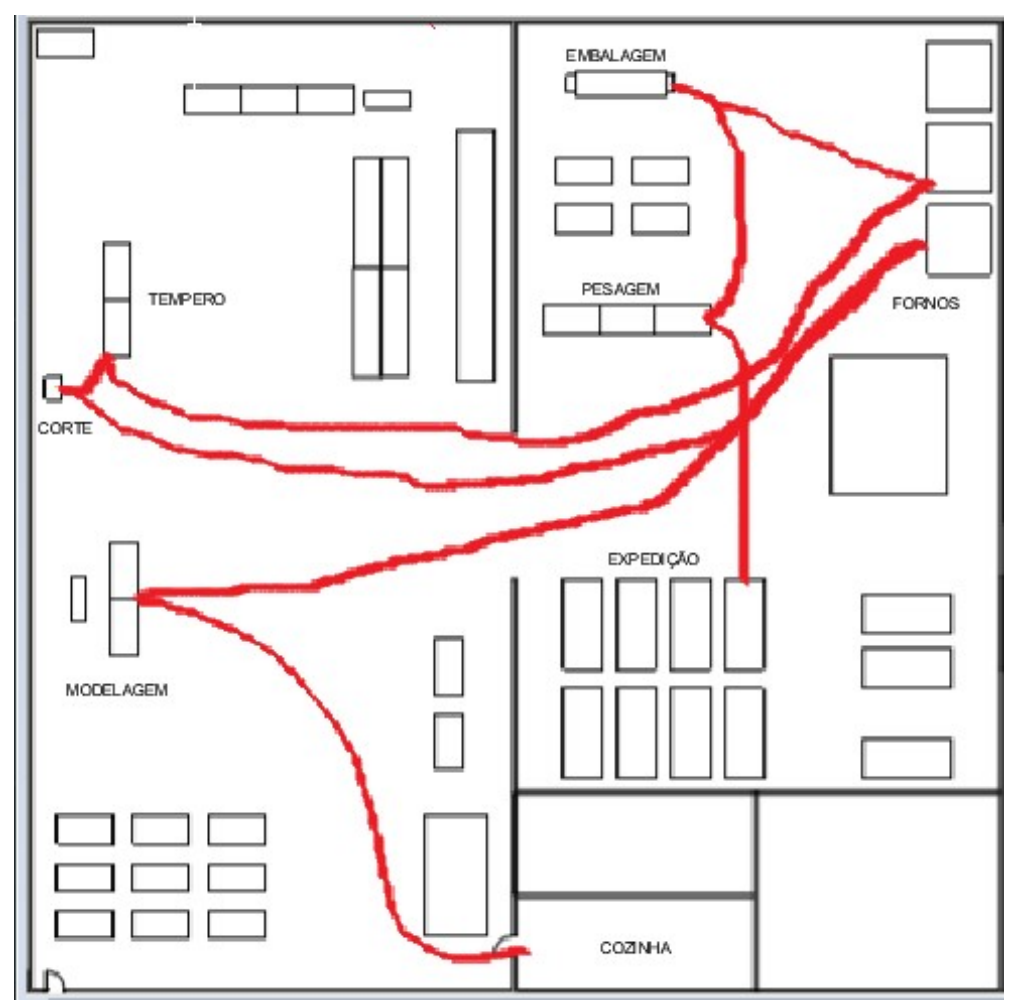

Fonte: Próprio autor (2020)

Com o desenho do percurso no diagrama e metragem das distâncias percorridas, pôdese perceber a inviabilidade dolayout, onde o processo não possuía um fluxo contínuo de produção e havia perdas por movimentação.

Foi analisado visualmente as células de modelagem, corte e tempero e identificou-se que estavam distantes, onde foi proposto a aproximação das etapas produtivas, gerando fluxo contínuo para as atividades realizadas.

A figura 02, apresenta o diagrama de espaguete após a alteração do layout,onde notase uma redução significativa da movimentação.

Figura 02: Diagrama de espaguete cenário após alteração do layout 


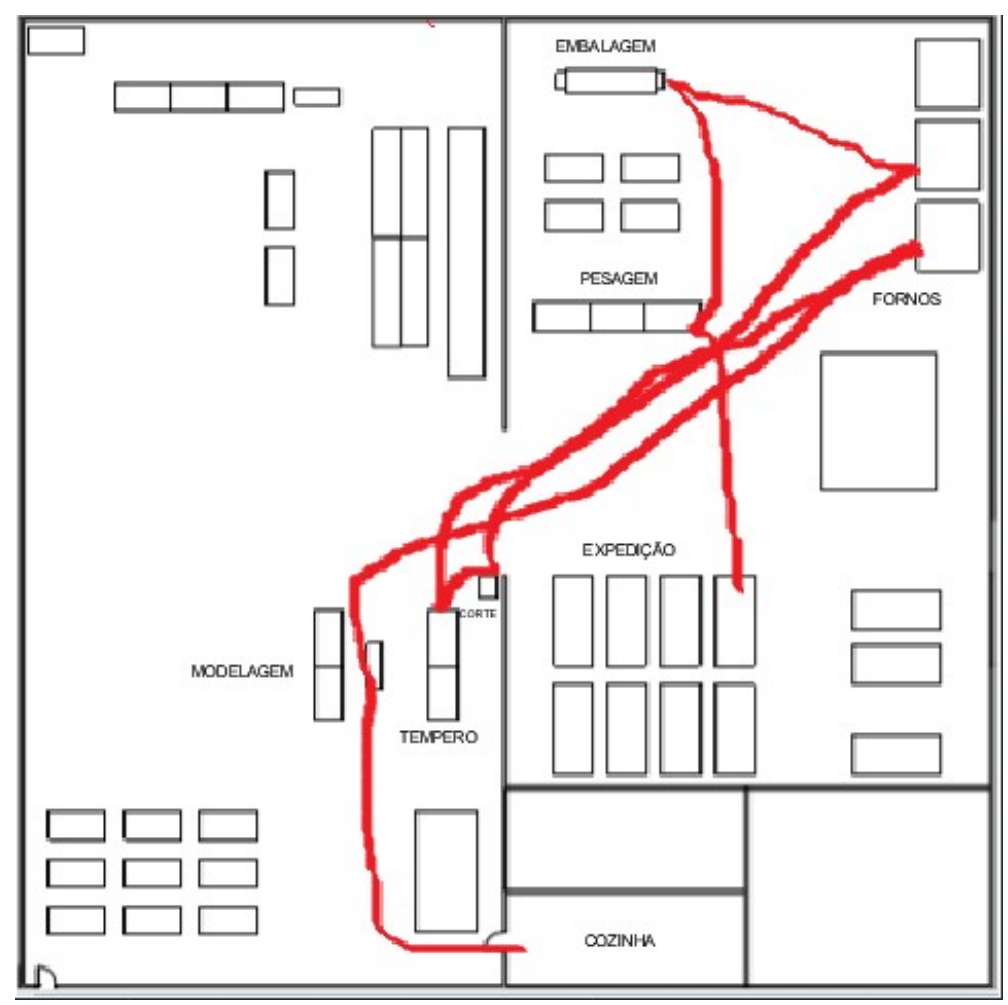

Fonte: Próprio autor (2020)

Os diagramas obtiveram respectivamente uma redução de 8.981 metros percorridos, através da aproximação das células de modelagem, corte e tempero,totalizando no cenário após alteração do layout 15.331 metros.

Com a alteração, conseguiu-se obter uma redução de 37\%de movimentação dos operadores entre as etapas, comprovando a eficiência da alteração do layout.

Em seguida, foram analisadas as etapas do processo produtivo. Através da simulação no software Arena, foi possível identificar 14 etapas no processo até o produto final, conforme figura 03.

Figura 03: Etapas de produção cenário primário

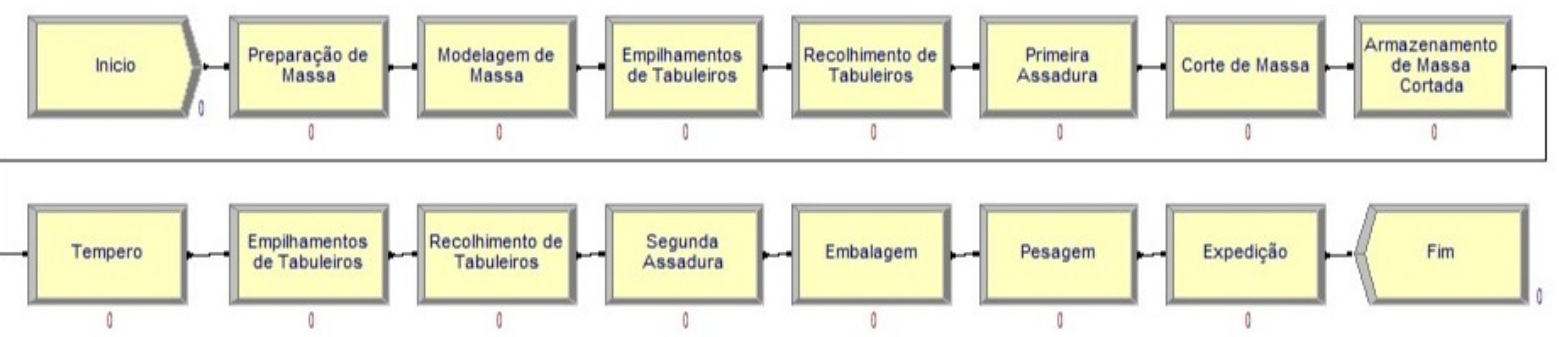


Fonte:Próprio autor (2020)

Ao aplicar o método Kaizen em busca de melhorias no processo, notou-se que as etapas de empilhamento e recolhimento de tabuleiros do processo correspondem a perca por processamento, ou seja, essas etapas não agregam valor e geram gargalos. Diante disso, foi realizado o estudo das perdas utilizando a ferramenta Lean Manufacturing, e ainda utilizando o software, foi modelado a simulação do processo, propondo a eliminação das etapas de empilhamento e recolhimento dos tabuleiros, que em seguida foi aplicado de forma práticaà produção. É possível visualizar o cenário após aplicação da metodologia na figura 02.

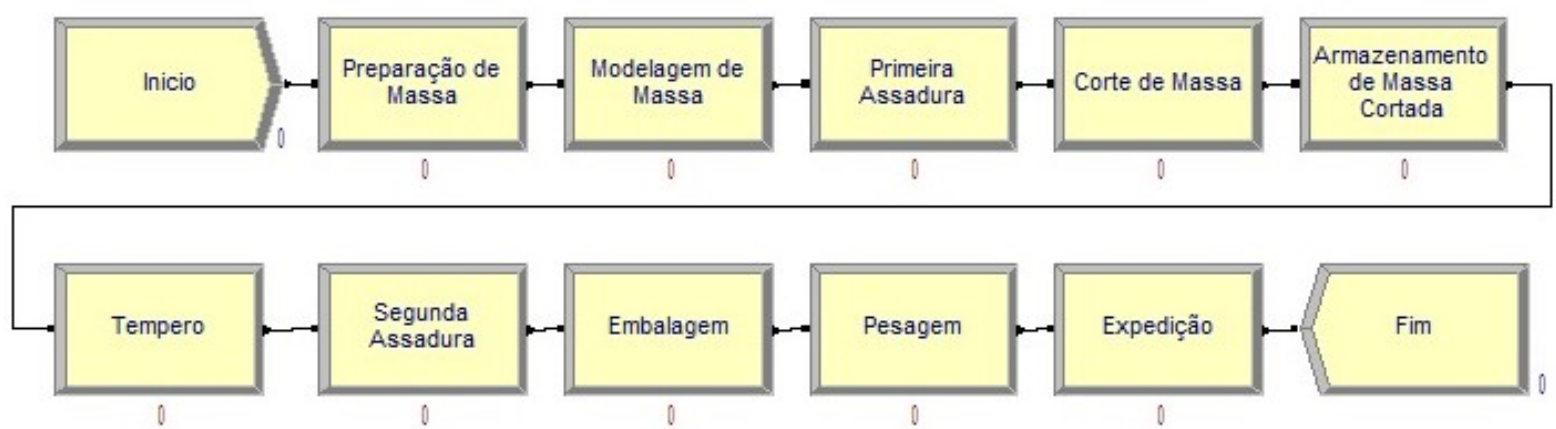

Figura 04: Etapas de produção após aplicação da ferramenta

Fonte:Próprio autor (2020)

A proposta eliminou as etapasde empilhamento e recolhimento de tabuleiros realizado pelas operadoras responsáveis pela modelagem e tempero, onde o forneiro passava recolhendo e adicionando-os ao carrinho para assadura. Com isto, as operadoras passaram a modelar e temperar já encaixando os tabuleiros diretamente no carrinho de assadura, agilizando o processo e reduzindo etapas do forneiro na operação.

Para uma melhor análise da redução das atividades, foi realizado na tabela 03 o levantamento do custo de produção no cenário primário para identificar o tempo de cada funcionário e seu custo nas atividades desenvolvidas no processo produtivo.

Tabela03: Custo de produção cenário primário 


\begin{tabular}{|c|c|c|c|c|c|c|}
\hline Membro 01 & $\mathrm{R} \$$ & 0,11 & Ajudante Confeiteiro & 2550 & $\mathrm{R} \$$ & 287,05 \\
\hline Membro 02 & $\mathrm{R} \$$ & 0,11 & Ajudante Confeiteiro & 2550 & $\mathrm{R} \$$ & 287,05 \\
\hline Membro 03 & $\mathrm{R} \$$ & 0,11 & Ajudante Confeiteiro & 2550 & $\mathrm{R} \$$ & 287,05 \\
\hline Membro 04 & $\mathrm{R} \$$ & 0,11 & Ajudante Confeiteiro & 2550 & $\mathrm{R} \$$ & 287,05 \\
\hline Membro 05 & $\mathrm{R} \$$ & 0,11 & Ajudante Confeiteiro & 2550 & $\mathrm{R} \$$ & 287,05 \\
\hline Membro 06 & $\mathrm{R} \$$ & 0,11 & Ajudante Confeiteiro & 2550 & $\mathrm{R} \$$ & 287,05 \\
\hline Membro 07 & $\mathrm{R} \$$ & 0,11 & Ajudante Confeiteiro & 2550 & $\mathrm{R} \$$ & 287,05 \\
\hline Membro 08 & $\mathrm{R} \$$ & 0,11 & Ajudante Confeiteiro & 562 & $\mathrm{R} \$$ & 63,26 \\
\hline Membro 09 & $\mathrm{R} \$$ & 0,11 & Ajudante Confeiteiro & 345 & $\mathrm{R} \$$ & 38,84 \\
\hline Membro 10 & $\mathrm{R} \$$ & 0,11 & Ajudante Confeiteiro & 42 & $\mathrm{R} \$$ & 4,73 \\
\hline Membro 11 & $\mathrm{R} \$$ & 0,11 & Ajudante Confeiteiro & 1275 & $\mathrm{R} \$$ & 143,53 \\
\hline Membro 12 & $\mathrm{R} \$$ & 0,14 & Confeiteiro & 2550 & $\mathrm{R} \$$ & 365,29 \\
\hline Membro 13 & $\mathrm{R} \$$ & 0,14 & Confeiteiro & 189 & $\mathrm{R} \$$ & 27,07 \\
\hline Membro 14 & $\mathrm{R} \$$ & 0,15 & Maceiro & 525 & $\mathrm{R} \$$ & 81,13 \\
\hline Membro 15 & $\mathrm{R} \$$ & 0,19 & Forneiro & 564,3 & $\mathrm{R} \$$ & 105,02 \\
\hline & & & & TOTAL & $\mathrm{R} \$$ & $2.838,22$ \\
\hline
\end{tabular}

Fonte:Próprio autor $(2020$

Com a aplicação da metodologia e redução das etapas do forneiro,é possível visualizar na tabela 04 que houve redução do custo de produção, e com a agilidade do processo.

Tabela 04: Custo de produção após implementação da metodologia

\begin{tabular}{|l|cc|c|c|c|c|}
\hline \multicolumn{9}{|c|}{ CUSTO PRODUÇÃ O DEPOIS } \\
\hline & \multicolumn{2}{|c|}{ Valor P/ MIN } & \multicolumn{2}{c|}{ Função } & Minutos trabalhados & \multicolumn{2}{c|}{ Custo produção } \\
\hline Membro 01 & $\mathrm{R} \$$ & 0,11 & Ajudante Confeiteiro & 2550 & $\mathrm{R} \$$ & 287,05 \\
\hline Membro 02 & $\mathrm{R} \$$ & 0,11 & Ajudante Confeiteiro & 2550 & $\mathrm{R} \$$ & 287,05 \\
\hline Membro 03 & $\mathrm{R} \$$ & 0,11 & Ajudante Confeiteiro & 2550 & $\mathrm{R} \$$ & 287,05 \\
\hline Membro 04 & $\mathrm{R} \$$ & 0,11 & Ajudante Confeiteiro & 2550 & $\mathrm{R} \$$ & 287,05 \\
\hline Membro 05 & $\mathrm{R} \$$ & 0,11 & Ajudante Confeiteiro & 2550 & $\mathrm{R} \$$ & 287,05 \\
\hline Membro 06 & $\mathrm{R} \$$ & 0,11 & Ajudante Confeiteiro & 2550 & $\mathrm{R} \$$ & 287,05 \\
\hline Membro 07 & $\mathrm{R} \$$ & 0,11 & Ajudante Confeiteiro & 2550 & $\mathrm{R} \$$ & 287,05 \\
\hline Membro 08 & $\mathrm{R} \$$ & 0,11 & Ajudante Confeiteiro & 562 & $\mathrm{R} \$$ & 63,26 \\
\hline Membro 09 & $\mathrm{R} \$$ & 0,11 & Ajudante Confeiteiro & 345 & $\mathrm{R} \$$ & 38,84 \\
\hline Membro 10 & $\mathrm{R} \$$ & 0,11 & Ajudante Confeiteiro & 42 & $\mathrm{R} \$$ & 4,73 \\
\hline Membro 11 & $\mathrm{R} \$$ & 0,11 & Ajudante Confeiteiro & 1275 & $\mathrm{R} \$$ & 143,53 \\
\hline Membro 12 & $\mathrm{R} \$$ & 0,14 & Confeiteiro & 2550 & $\mathrm{R} \$$ & 365,29 \\
\hline Membro 13 & $\mathrm{R} \$$ & 0,14 & Confeiteiro & 189 & $\mathrm{R} \$$ & 27,07 \\
\hline Membro 14 & $\mathrm{R} \$$ & 0,15 & Maceiro & 525 & $\mathrm{R} \$$ & 78,75 \\
\hline Membro 15 & $\mathrm{R} \$$ & 0,19 & Forneiro & 329,3 & $\mathrm{R} \$$ & 62,57 \\
\hline
\end{tabular}

Fonte: Próprio autor (2020) 
Por fim, o custo de produção obteve respectivamente uma redução de 1,58\%, comprovando a eficiência da aplicação do Lean Manufacturing, resultando na redução das atividades.

\section{CONCLUSÃO}

O trabalho realizado buscou identificar desperdícios de movimentação nos processos realizados na linhade produção da torradinha alho, assim, propondo redução dos mesmos através da aplicação da metodologia Lean Manufacturing.

Através da coleta de dados, foi identificado que os operadores percorriamuma distância de 24.312 metros entre as etapas de produção. Com a mudança do layoutposicionando a linha de produção em sequência, obteve-se novos valores, nos quais, houveredução de $37 \%$ de movimentação. Contudo, após as reduções com aalteração de layout, foi possível obter uma redução de $1,58 \%$ do custo de produçãocom aaplicação do método Lean Manufacturing.

\section{REFERÊNCIAS BIBLIOGRÁFICAS}

ARUNAGIRI, P., \& GNANAVELBABU, A. (2014). Identification of High Impact Lean Production Tools in Automobile Industries using Weighted Average Method. Procedia Engineering, 97, 2072-2080.

BEZERRA, L. Utilizando o software Microsoft Excel na abordagem dos conceitos iniciais de função do $1^{\circ}$ e $2^{\circ}$ grau. Paraíba, 2019

CASTRO, Diogo Amorim. Computação Gráfica Aplicada a Engenharia: Estudo de Caso do Laboratório de Engenharia da Computação (UEFS). 2010. 79f. Trabalho de Conclusão de Curso (Graduação em Engenharia Civil) - Universidade Estadual deFeira de Santana, Feira de Santana, 2010.

FREITAS, E. B. Diagrama de Espaguete. Engenharia de Produção, v 5, 2013

LINHAR, Adriano. LUZZATTO, Elquier Smaniotto. Um protótipo de um sistema paradeterminação da capacidade produtiva instalada com base em estudos decronoanálise industrial para pequenas empresas. 2011. 98 f. Monografia(Tecnólogo em Análise e 
Desenvolvimento de Sistemas) - Universidade TecnológicaFederal do Paraná, Campus Pato Branco, Pato Branco, 2011.

OHNO, T. Sistema Toyota de Produção - Além da Produção em Larga Escala, Porto Alegre, Editora Bookman, 1997.

PRADO, D. Usando o Arena em Simulação. Belo Horizonte: Editora de Desenvolvimento Gerencial, 1999.

TAYLOR, F. W. Princípios de administração científica. 7. ed. São Paulo: Atlas, 1982.

TMB Consulting Group. Apostila para Treinamento de Kaizen Chão de Fábrica. São Paulo, 2000 
ANEXO 01 


\section{Autorização de apresentação de dados da pesquisa}

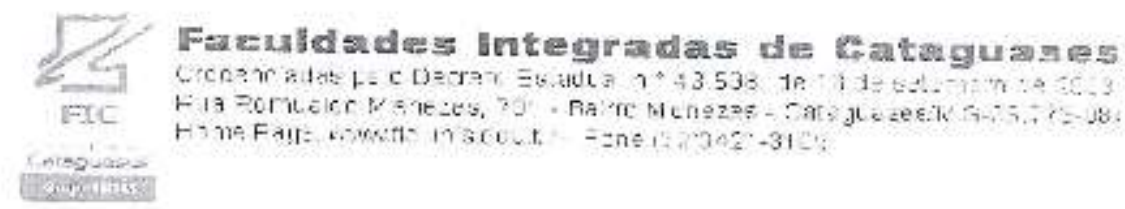

\section{CARTA PARA AUTORTZACAO DE PESQUISA}

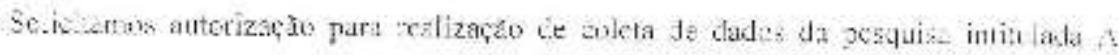

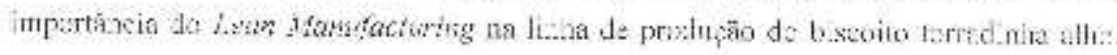

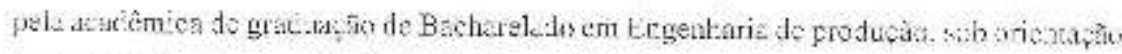

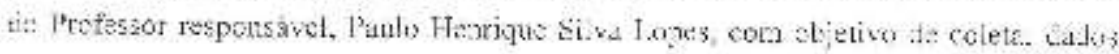

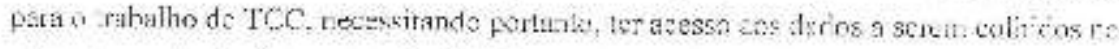

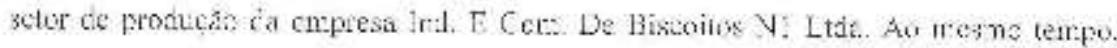

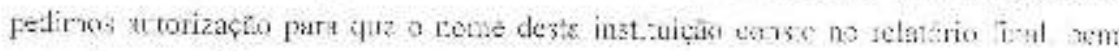

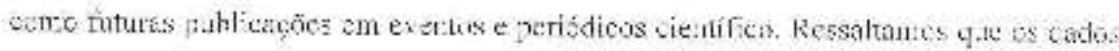

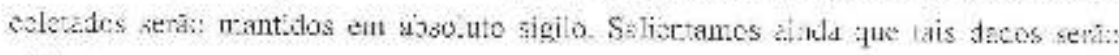

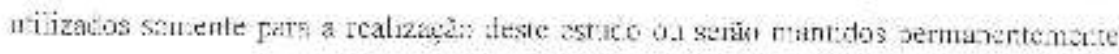

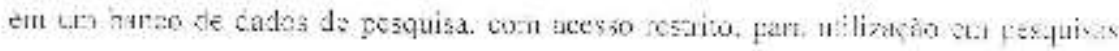

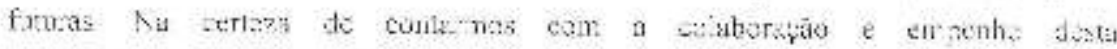

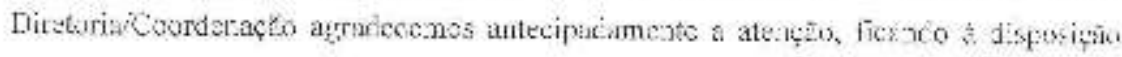
pa:a quaisquer exclatcintentos aiviorsis cue se tizeror- nccossáras.

Caguziss-mi, ol de 10 ce 2020

1). Conosidanus cur a solcitacao

1: Who eveorcamos cum a soliciacto

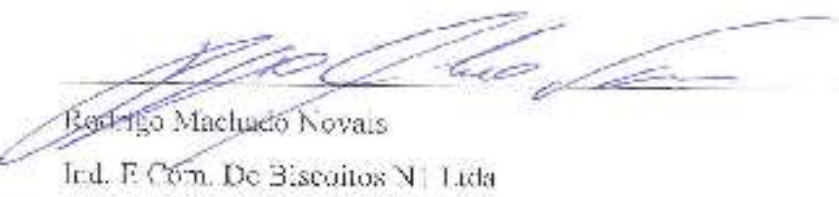

